\title{
Team working: the key to implementing guidelines?
}

Health care delivery is a complex organisational process. Success depends on the coordination of the work of all members of the team as well as on the competence of individuals. Planning care and team working are vital components of quality improvement initiatives if improvement in practice is to be achieved. Despite the complexity of the process and the use of advanced technology most clinical interventions are routine procedures. So, it is possible to make clear statements that connect important technical aspects of care with significant organisational information - that is, to make explicit the clear link between what is to be done, how it is to be done, by whom, and when. Such a statement is a multidisciplinary guideline. It should encompass the sort of information that is included in medical guidelines and other professional care plans and be set in the context of a clear organisational framework and be shared by all members of the multidisciplinary team.

\section{Resistance to implementing guidelines}

Much resistance and many obstacles have been put in the path of the uptake and use of guidelines. ${ }^{1}$ Some say they limit clinical freedom or stifle innovation. Others are concerned about the potential to formalise unsound practice and have anxieties about exposure of negligence. In our view these objections have no particular substance. Some individual freedom will be sacrificed for the benefits of team working, but that sacrifice is small. Senior clinicians are key individuals in agreeing guidelines and recognise areas where there is a true need for discretion and decision making. The restriction of clinical freedom is trivial. Guidelines do not stifle innovation: they prevent one off experimentation on patients and provide a framework which should allow measurement of the effect of the total package of care on outcome. Guidelines formulated in full, open discussion are unlikely to formalise unsound practice. Rather, by making practice explicit they should bring about its improvement. Anxieties about legal challenges seem ill founded except for those who wish to work in a totally individualistic way. The existence of an explicit and open standard of care is a protection for patient and practitioner rather than a source of difficulty.

The real source of the problems with the integration of guidelines in practice lies elsewhere. Despite the widely held belief that professionals in the health service work well as teams the reality shows a different picture. ${ }^{2}$ Each profession plans and documents its care separately, and this separation is often carried over into the way that each works. The current model of care is dominated by the medical paradigm which treats each new case as a new episode, multidisciplinary care planning is the exception rather than the rule, and guidelines that "medical staff" will follow are still emphasised. ${ }^{1}$ For doctors the real issue is whether they wish to be members of a team or continue as individualistic operators. The personality traits of those drawn to a career in medicine have been described in the following terms. "On the whole they are people with a strong need to work autonomously, they need to analyse a problem and then chart their own course of action. They don't have the literacy of team work, collaboration, and empowering other people. They are used to personal intervention rather than working in a team." 3

The onus of implementing medical guidelines has been given covertly to junior doctors. But within the medical hierarchy these doctors are usually expected to be developing their own personal skills, concentrating on professional examinations, and responding primarily to their medical teams rather than working explicitly as part of a multidisciplinary group. Clinical guidelines developed by any institution need the commitment of people working for that institution. Doctors in training do not have the necessary investment in organisations for which they work for only a very short time. Guidelines which have a medical bias do not encompass the work of other professionals, and the resulting lack of sense of ownership is an important contributory factor in the failure of guidelines to work. Without that sense of ownership clinical guidelines will remain in the coat pockets of staff or lie somewhere gathering dust. ${ }^{45}$

\section{Conscious team building}

By developing guidelines that combine the usually separate plans of each profession it is possible to create a unitary plan incorporating known national and local guidelines. Part of such a process must be a conscious attempt at team building, with group working as the way to bring together the differing professional attitudes. ${ }^{6}$ Bringing together staff to discuss individual contributions will allow recognition of the part played by colleagues in care delivery, show where there is overlap and repetition of work, and highlight the need for mutual dependence. Consultant medical staff have a central role in bringing the work of all doctors into the multiprofessional arena. As multidisciplinary protocols come into use, the benefits become apparent: reduced documentation, a more efficient process, and a tool for clinical audit. Uniprofessional patient notes are an important expression of a unified team approach to care.

In our experience multidisciplinary guidelines are much more likely to be implemented and accepted than medical guidelines, and they become readily accepted once the hurdles of getting people to discuss their work in groups and to work in teams are overcome. But there are problems with devising such guidelines. One is the belief that each step should have been the subject of verification. Such standards are not available for many aspects of routine care. The total package of care as normally given is so unstandardised that proof of the value of the total package is simply not available. Of course, proof is available to support the value of some single stages of care, and guidelines should incorporate proven best care. Evaluation of the merits of protocols of care will be possible only when there are enough protocols to permit valid comparison. Meanwhile, lack of proof that planned, team care is better than unplanned care given by uncommunicative individuals is 
no reason for not adopting planned approach. Moreover, analogies from other complex production processes support strongly the view that adopting protocols of care will improve the quality of practice.

Medical guidelines are unlikely to have an impact on clinical care for the very reason that they are medical and are not shared by all members of a clinical team. The difficulties encountered in simply disseminating guidelines suggests that the problem lies with the organisation of care. We are trapped into working within professional boundaries. Until we come to grips with the reality of the need to improve coordination and planning of care and to learn to work within multiprofessional teams, guidelines are unlikely to have any impact. Uniprofessional guidelines are unlikely to work. Multiprofessional guidelines may be the key to unlock the potential for change in health care.
MARTIN McNICOL

Chair

AMANDA LAYTON

Protocol Development Coordinator

GRAHAM MORGAN

Senior Nurse Advisor

Central Middlesex Hospital NHS Trust, London NW10 7NS

1 Delamothe $T$. Wanted: guidelines that doctors follow. $B M \mathcal{F}$ 1993;307:218

Morgan G. The implication of patient focused care. Nursing Standard 1993;52:37-9.

3 Smith R. Leadership and doctors. BMF 1992;305:137-8.

4 Gabbay J, Layton AJ. Evaluation of audit of medical records in a district general hospital. Quality in Health Care 1992;1:43-7.

Bell D, Layton AJ, Gabbay J. Use of a guideline based questionnaire to audit hospital care of acute asthma. BMF 1991;302:1440-3

6 Firth-Cozens J. Building teams for effective audit. Quality in Health Care 1992;1:252-5.

7 Operational restructuring - a recipe for success. Health Care Viewpoint. London: Booz Allen Hamilton, 1988 . 